\title{
论文
}

\section{基于CryoSat-2卫星测高数据的北极海冰厚度 变化研究}

\author{
肖峰, 张胜凯, 李佳星, 耿通, 宣越, 李斐* \\ 武汉大学中国南极测绘研究中心, 武汉 430079 \\ *通讯作者, E-mail: fli@whu.edu.cn
}

收稿日期: 2020-10-23; 收修改稿日期: 2021-02-26; 接受日期: 2021-04-26; 网络版发表日期: 2021-05-18

国家重点研发计划项目(编号: 2017YFA0603104)、国家自然科学基金项目(批准号: 41531069、41706216)和测绘遥感信息工程国家重点实验 室专项科研经费资助

\begin{abstract}
摘要 北极海冰是地球气候系统的重要因子, 获取精确的海冰厚度及其变化信息对于开展北极和全球变化研究 等有着重要的意义. 卫星测高是获取连续、大范围海冰厚度的主要方法之一. 冰间水道识别是卫星测高方法估算 海冰厚度的关键之一. 基于CryoSat-2数据，利用遥感影像对两种主要的冰间水道识别方法进行了对比，发现波形 特征法能够更好地识别冰间水道. 考虑到雷达信号对海冰表面积雪的不完全穿透, 对海冰干舷-厚度转化模型进 行了优化, 通过选取合适的输入参数, 获取了2010年11月至2019年12月北极海冰厚度, 并利用IceBridge海冰厚度 产品和仰视声呐数据对计算结果进行了验证, 结果表明本文海冰厚度解算精度优于 $0.2 \mathrm{~m}$. 最后, 结合PIOMAS海 冰模式数据、北极气温和海表面温度数据对北极海冰厚度变化特征进行了分析，发现2014年北极海冰厚度出现 剧烈增长的现象.
\end{abstract}

关键词 CryoSat-2, 北极海冰, 厚度, 全球变化

\section{1 引言}

作为地球冰冻圈的重要组成部分，北极海冰历来 是全球变化研究的热点之一(秦大河等，2014; 市林根 等，2016). 海冰隔绝了海洋和上层大气之间的直接热 量传输, 影响着全球辐射平衡, 进而在全球气候变化中 起着重要的作用(Screen和Simmonds, 2010). 卫星观测 资料表明, 在过去 30 余年, 北极海冰分布和范围发生了 较大的变化(Ricker等, 2017). 海冰厚度是获取海冰物质 平衡的关键参数, 同时也是最难观测的海冰参数之一.
目前, 用于海冰厚度观测方法主要包括钻孔观 测、仰视声呐、电磁感应、微波遥感以及卫星测高 等. 其中, 钻孔观测精度最高, 但是受限于海冰区的作 业环境, 很难进行大量观测. 仰视声呐和电磁感应方法 能够获得局部地区、一定时间段内的海冰厚度信息， 但难以进行大范围内连续观测. 微波遥感和卫星测高 手段可以获得连续、大范围内的海冰厚度信息，但是 微波遥感方法仅适用于薄冰区域的观测. 卫星测高方 法能够连续获取全球范围内的海冰厚度信息, 被广泛 应用于海冰厚度研究中. 
利用卫星测高估算海冰厚度的方法最早由Laxon 等(2003)提出, 他利用ERS- $1 / 2$ 数据获取了 $65^{\circ} \mathrm{N} \sim 81.5^{\circ} \mathrm{N}$ 的北极海冰厚度, 通过与潜艇声呐数据对比, 验证了 卫星测高估算海冰厚度的可靠性. 随后卫星测高方法 被广泛应用于海冰厚度估算中(Giles等，2008; Kwok 等，2009；Laxon等，2013；Tilling等，2015，2016; Ricker等，2017). 卫星测高方法估算海冰厚度时首先 利用测高数据区分海冰与相邻冰间水道, 计算出海冰 干舷, 然后根据流体静力学浮力定律, 建立海冰干舷厚度转化方程, 最后结合相应的参数(积雪深度和密 度、海冰密度、海水密度等), 得到海冰厚度. 最初, Laxon等(2003)利用ERS-1/2估算海冰厚度时，通过分 析海冰表面和冰间水道回波波形特征, 利用脉冲峰值 (PP)来识别冰间水道. 在此基础上，Laxon等(2013)基 于CryoSat-2数据，利用高斯模型拟合回波波形，利用 PP值和脉冲累计标准差(SSD)来识别冰间水道. Zwally 等(2008)、Kwok和Cunningham(2008)基于海面高程总 是低于海冰表面高程的假设, 提出将一定范围内相对 高程最低的部分点的平均高程视为该范围内的冰间水 道高程. Kurtz等(2009)利用ICESat数据估算海冰厚度 时, 借助机载雷达影像进行了冰间水道的识别. 对海 冰干舷-厚度转化模型中输入参数的取值, 各个学者使 用的数据来源也不尽相同. 如对于海冰表面积雪深度, Laxon等(2003)采用的是北极地区实测的月度气象资 料(W99模型, Warren等, 1999)的积雪深度值; Zwally等 (2008)根据一年冰和多年冰表面积雪深度的不同，分 别采用AMSR-E和W99积雪深度值；Laxon等(2013)在 解算中, 对一年冰表面积雪深度值采用W99估值的一 半. 此外, 现有研究在利用雷达测高卫星估算海冰厚 度时，一般认为雷达信号对海冰表面积雪完全穿透， 然而研究表明, 雷达信号并不能对积雪完全穿透, 其 穿透深度与信号频率、积雪湿度和温度相关(Ricker 等, 2014), 这便导致最后的海冰厚度估算结果出现 偏差.

本文的主要目的是利用CryoSat-2数据, 通过对比 不同冰间水道识别方法，计算海冰干舷，考虑雷达信 号的不完全穿透性，优化海冰干舷-厚度转化模型，选 取合适的模型参数, 获取2010 2019年较长时间尺度的 北极海冰厚度, 并利用航空测量和仰视声呐数据对其 进行验证, 最后结合海冰模式和气象数据, 对北极海 冰厚度变化特征进行讨论分析.

\section{2 研究数据}

\subsection{CryoSat-2数据}

CryoSat-2卫星发射于2010年，其主要目的是对全 球冰盖和海冰进行监测, 获取其变化趋势(Wingham 等, 2006). CryoSat-2卫星搭载了新型合成孔径/干涉雷 达高度计(synthetic aperture radar interferometric radar altimeter, SIRAL), 该高度计综合了SAR和干涉技术的 优点, 能够大大提高其对冰盖边缘和海冰观测的精度 (肖峰等, 2020). SIRAL有三种观测模式, 其中低分辨 率模式(low resolution mode, LRM)主要用于内陆冰盖 和海洋的观测, SARIn模式(synthetic aperture radar interferometry mode)主要用于冰盖边缘、小型冰山和 内陆冰川的观测, SAR模式(synthetic aperture radar mode)主要用于海冰观测.

考虑到CryoSat-2卫星的覆盖范围, 并与前人研究 保持一致(Laxon等, 2013; Tilling等, 2018), 本文所选 的研究区域为 $65^{\circ} \mathrm{N}$ 以北, $88^{\circ} \mathrm{N}$ 以南的海域. 在 2014 年 9 月之前, CryoSat-2卫星采用SAR和SARIn模式进行海 冰观测, 2014年9月之后, CryoSat-2卫星主要采用SAR 模式进行海冰观测. 因此, 本文主要采用基线 $\mathrm{C}$ 版本的 CryoSat-2 SAR和SARIn模式数据获取北极海冰厚度, 该数据包含了卫星轨道与时间信息, 雷达回波测量信 息、波形统计信息及仪器与外部校正信息.

\subsection{IceBridge海冰厚度产品}

为了填补ICESat卫星(2003 2009年)和ICESat-2卫 星(于 2018 年发射)之间的数据间断, NASA于 $2007 / 08$ 国际地球年期间, 开始启动了 IceBridge(冰桥)计划, 利 用航空飞机监测两极冰盖、冰架和海冰的变化. 利用 IceBridge计划中的激光测高数据和雪雷达数据, 可以 计算得到航线上的海冰厚度信息. 美国雪冰数据中心 (National Snow and Ice Data Center, NSIDC)提供了 IceBridge海冰厚度产品(Kurtz等, 2013). 利用该产品对 CryoSat-2海冰厚度估算结果进行验证.

\section{3 仰视声呐数据}

为探究北冰洋流域淡水含量, 美国伍兹霍尔海洋 学研究所开展了波弗特环流计划(Beaufort Grye Exploration Project, BGEP). 该计划通过在波弗特海域安 装多种传感器测量设备, 监测海水温度、盐度、流速 
以及海冰吃水深度 (Kemp等, 2005). 其中, 海冰吃水深 度是利用安装在水下的仰视声呐仪(upward looking sonar，ULS)测量得到的. BGEP在波弗特海域共安装 了四个仰视声呐仪, 位置如图1所示. 四个仰视声呐仪 的工作时段如表1所示. 其中，仰视声呐仪 C只工作到 2008年8月，因此本文选择A、B、D三个仰视声呐仪 的数据用于结果验证.

\section{3 计算方法}

\section{1 冰间水道识别}

利用卫星测高估算海冰厚度时，冰间水道的识别 最为关键. 常用的冰间水道识别方法主要有三种: 遥 感影像法、最低点法和波形特征法. 遥感影像法利用 与测高数据同步的卫星影像来区分海水和海冰，识别 精度最高，但不适合大范围内的解算. 最低点法假设 一定范围内海冰表面高程大于海面高程，选择该范围 内高程最低的高程点为冰间水道. 波形特征法利用测 高波形特征参数进行冰间水道的识别, 常用的参数主 要有 PP(pulse peakiness)和 SSD(stack standard deviation)等.

图2和图3分别是利用最低点法和波形特征法进行

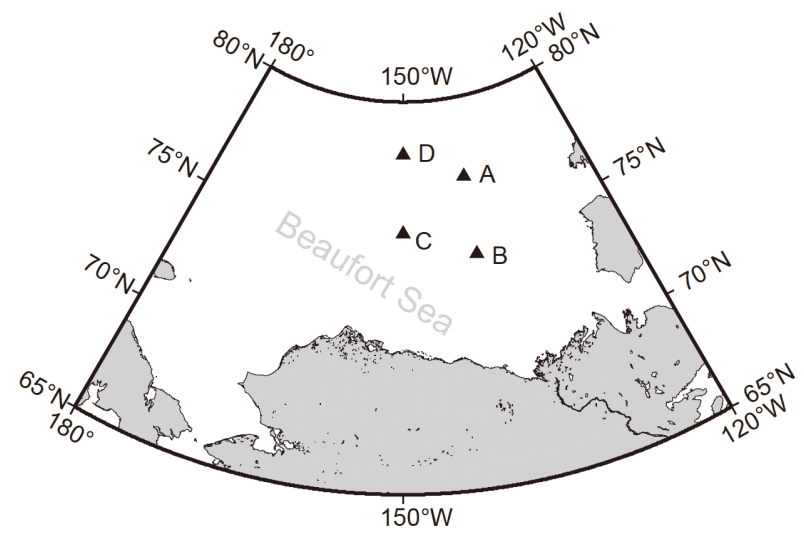

图 1 波弗特环流计划仰视声呐安装位置

表 1 四个仰视声呐仪工作时段

\begin{tabular}{cc}
\hline 仰视声呐仪 & 观测时段 \\
\hline A & $2003-08-14 \sim 2018-09-24$ \\
B & $2003-08-24 \sim 2018-09-24$ \\
C & $2003-08-27 \sim 2008-08-09$ \\
D & $2005-08-28 \sim 2018-09-24$ \\
\hline
\end{tabular}

冰间水道提取的结果, 并与MODIS影像进行了对比. 实验选取的CryoSat-2卫星地面轨迹全长约为 $176 \mathrm{~km}$, CryoSat-2数据的采集时间与MODIS影像的采集时间 小于 10 个小时. 在利用最低点法提取冰间水道时, 参考 Yi等(2011)的算法, 沿轨每 $20 \mathrm{~km}$ 作为一个独立窗口, 选 择最小的三个点的平均高程作为该窗口内冰间水道的 高程值. 在波形特征法中, 选取 PP $>18$ 且 $\mathrm{SSD}<6.29$ 的星 下点作为冰间水道(Tilling等, 2018). 对比最低点法和 波形特征法的实验效果，最低点法的识别精度与所选 窗口的长度有关，如对于 $20 \mathrm{~km}$ 窗口内没有冰间水道 (图2b)或者只有一个高程点位于冰间水道上(图2c)时, 最低点法将剩余高程较低的海冰误判为冰间水道, 使 得冰间水道的高程值被高估，进而导致了海冰干舷高 度值出现误差. 相比而言, 波形特征法的识别精度更 高，因此本文在后续计算中，选用波形特征法识别冰 间水道.

\section{2 海冰干舫提取}

海冰干舷高度 $\left(h_{\mathrm{fb}}\right)$ 可根据测高卫星观测的冰面高 程值和瞬时海面高程求得(Ricker等, 2014):

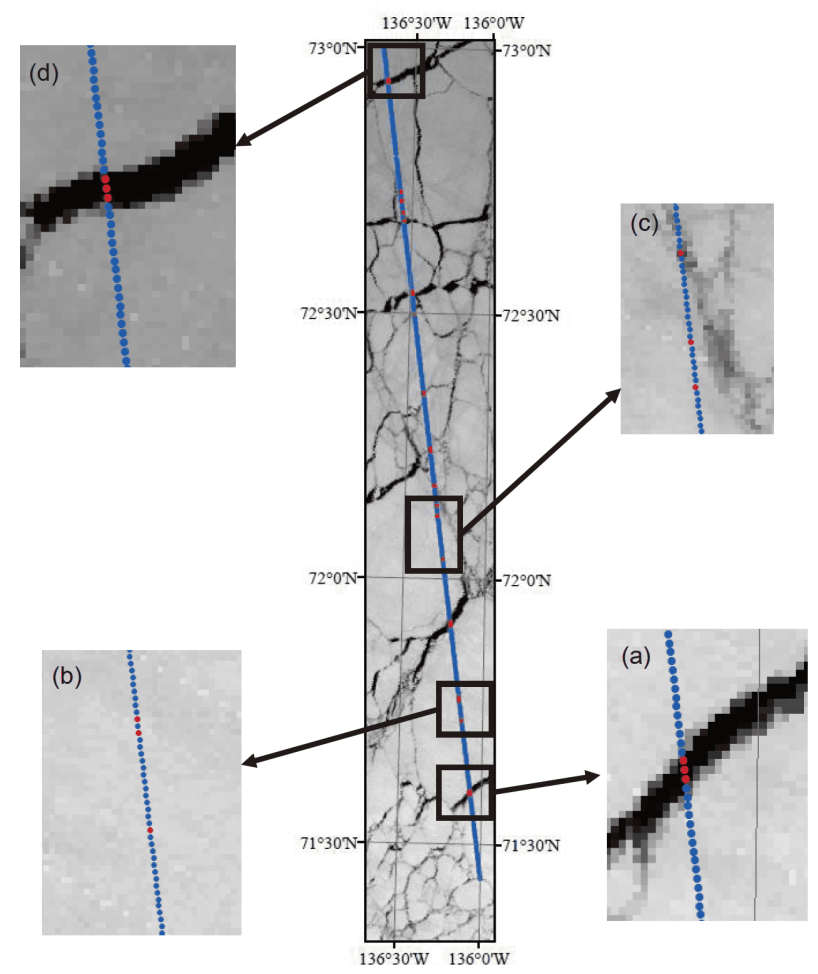

图 2 利用最低点法提取冰间水道结果 


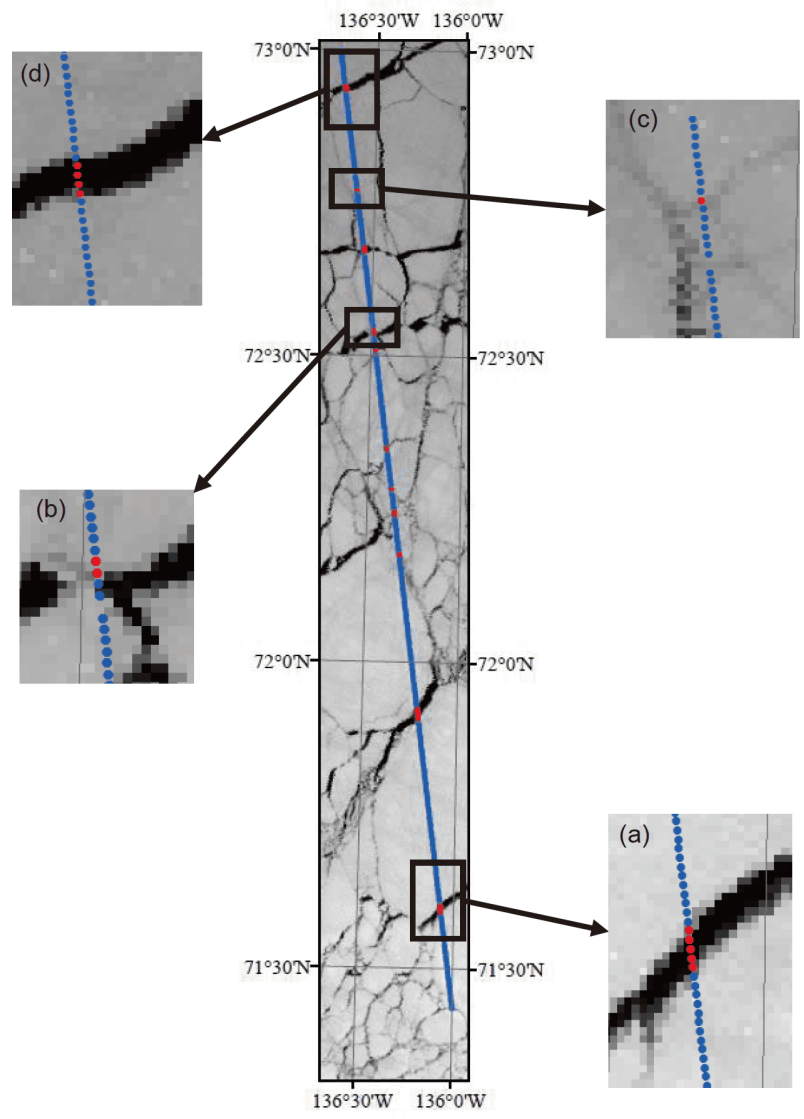

图 3 利用波形特征法提取冰间水道结果
过以下公式计算(Ricker等, 2014):

$h_{\mathrm{si}}=\frac{\rho_{\mathrm{sw}}}{\rho_{\mathrm{sw}}-\rho_{\mathrm{si}}} h_{\mathrm{fb}}+\frac{\rho_{\mathrm{s}}}{\rho_{\mathrm{sw}}-\rho_{\mathrm{si}}} h_{\mathrm{s}}$,

式中, $h_{\mathrm{si}}$ 为海冰厚度; $\rho_{\mathrm{sw}} 、 \rho_{\mathrm{si}} 、 \rho_{\mathrm{s}}$ 分别表示海水密度、 海冰密度和积雪密度; $h_{\mathrm{s}}$ 为海冰表面积雪深度.

然而, 研究表明雷达信号的穿透界面通常在雪层 中(Willatt等, 2011), 即雷达信号不是总能完全穿透海 冰表面积雪. 之前研究中, 通常默认雷达信号对积雪完 全穿透(Ricker等, 2014; Tilling等, 2018). 如图4所示, 假 设雷达信号对海冰表面积雪的穿透深度为 $h_{\mathrm{ps}}$, 则根据 流体静力学浮力定律, 海冰干舷-厚度转化方程应为

$$
\begin{aligned}
h_{\mathrm{si}} & =\frac{\rho_{\mathrm{sw}}}{\rho_{\mathrm{sw}}-\rho_{\mathrm{si}}} h_{\mathrm{fb}}+\frac{\rho_{\mathrm{s}}-\rho_{\mathrm{sw}}}{\rho_{\mathrm{sw}}-\rho_{\mathrm{si}}} h_{\mathrm{s}}+\frac{\rho_{\mathrm{sw}}}{\rho_{\mathrm{sw}}-\rho_{\mathrm{si}}} h_{\mathrm{ps}} \\
& =\frac{\rho_{\mathrm{sw}}}{\rho_{\mathrm{sw}}-\rho_{\mathrm{si}}} h_{\mathrm{fb}}+\frac{\rho_{\mathrm{s}}-\rho_{\mathrm{sw}}}{\rho_{\mathrm{sw}}-\rho_{\mathrm{si}}} h_{\mathrm{s}}+\frac{\rho_{\mathrm{sw}}}{\rho_{\mathrm{sw}}-\rho_{\mathrm{si}}} \theta \cdot h_{\mathrm{s}},
\end{aligned}
$$

式中, $\theta$ 为雷达信号对海冰表面积雪的穿透率.

由公式(5)可知, 在海冰干舷-厚度转化过程中, 需 要用到海冰表面积雪深度及密度、海水密度、海冰密 度以及雷达信号对积雪的穿透率等参数. 然而, 由于北 极地区特殊的气候条件, 上述几个参数值的获取较为 困难. 在现有研究中, 一般采用经验参数或者模型拟 合值进行计算. 其中, 海水密度采用 $1024 \mathrm{~kg} \mathrm{~m}^{-3}$. 积雪

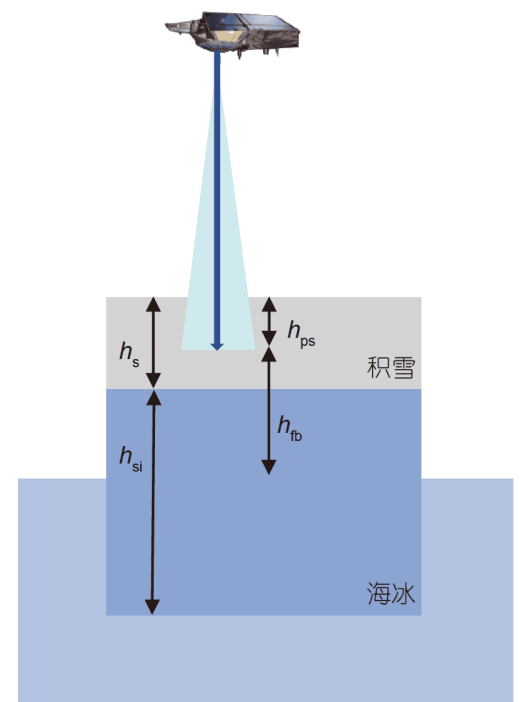

\section{3 海冰干舷-厚度转化模型优化和参数选取}

在雷达信号对海冰表面积雪完全穿透的假设下， 根据流体静力学浮力定律, 海冰干舷-厚度转化可以通

式中, $h_{\mathrm{ssh} 1}$ 为星下点的瞬时海面高程, 可通过星下点的 平均海平面高 $h_{\mathrm{mss} 1}$ 和海面高程异常 $h_{\mathrm{ssha}}$ 计算得到. 星下 点的平均海平面高可由CryoSat-2 L2数据产品获得.

在冰间水道上空, 卫星测高观测到的高程值 $h_{2}$ 为 星下点对应的瞬时海面高, 即

$$
\begin{aligned}
h_{2} & =h_{\mathrm{ssh} 2} \\
& =h_{\mathrm{ssha}}+h_{\mathrm{ms} 22} .
\end{aligned}
$$

因此, 海冰干舷高度计算公式(1)可以转换为

$$
\begin{aligned}
h_{\mathrm{fb}} & =h_{1}-\left(h_{2}-h_{\mathrm{mss} 2}+h_{\mathrm{mss} 1}\right) \\
& =h_{1}-h_{\mathrm{mss} 1}-\left(h_{2}-h_{\mathrm{mss} 2}\right) .
\end{aligned}
$$


深度和密度采用W99模型(Warren等，1999)拟合值，但 是由于W99模型是基于北极多年冰上的观测结果获得 的，对于一年冰上的积雪深度拟合较差(Laxon等, 2013), 因此一年冰上的积雪深度取值为W99模型值的 一半. 由于一年冰和多年冰的密度差异较大(Alexandrov等, 2010), 在解算中一年冰的密度采用 $917 \mathrm{~kg} \mathrm{~m}^{-3}$, 多年冰的密度采用 $882 \mathrm{~kg} \mathrm{~m}^{-3}$. 雷达信号对积雪的穿透 率与积雪的湿度、粒径等因素有关，最大的穿透深度 达到 $23 \mathrm{~cm}$ (Ricker等, 2014). Armitage和Ridout(2015)利 用IceBridge数据对CryoSat-2信号的穿透率进行了研 究, 发现对于一年冰, CryoSat-2雷达信号能够穿透 $96 \%$ 的雪层，而在多年冰上，穿透率为 $82 \%$. 在区分一年冰 和多年冰时，采用了挪威气象研究所发布的全球海冰 分类数据(Breivik等, 2012). 该数据集基于SSMIS亮温 数据和ASCAT背向散射值进行海冰分类，时间分辨率 为 1 天, 空间分辨率为 $10 \mathrm{~km}$.

\section{4 北极海冰厚度估算结果与精度验证}

\section{1 北极海冰厚度估算结果}

根据上述解算方法, 对2010年11月至2019年12月 期间的北极海冰厚度进行了估算. 由于夏季期间北极 海冰表面存在融池，影响了海冰厚度估算精度，因此 本文没有给出每年5 9月的海冰厚度估算结果. 图5为 2018年10月至2019年4月期间北极海冰月平均厚度的 空间分布, 图6为2018年10月至2019年4月期间北极海 冰月平均厚度的变化情况. 总体来看, 10 月份, 海冰分 布范围最小, 平均厚度约为 $1.544 \mathrm{~m}$, 仅在加拿大北极 群岛北部海域出现少量厚冰. 11月, 更多的厚冰出现 在格陵兰岛和加拿大北极群岛北部海域, 而在楚科奇 海、东西伯利亚海、拉普捷夫海和喀拉海等海域开 始出现薄冰, 平均海冰厚度约为 $1.511 \mathrm{~m} .12$ 月, 海冰范 围进一步扩大，平均厚度达到 $1.543 \mathrm{~m}$. 11月和 12 月海 冰厚度值略低于 10 月份海冰厚度值, 主要是因为 11 月 和12月出现了较多的薄冰, 使得整体海冰厚度值降 低. 1月, 海冰厚度继续增长, 在北极中央海域开始出 现大量厚冰, 平均海冰厚度约为 $1.602 \mathrm{~m} .2$ 月, 海冰厚 度出现剧烈增长, 平均厚度达到 $1.831 \mathrm{~m}$, 并在波弗特 和楚科奇海域出现了少量厚冰. 3 月, 海冰厚度继续增 长, 平均厚度约为 $2.043 \mathrm{~m}$, 同时更多的厚冰出现在各 个海域. 4 月份, 海冰厚度达到最大, 约为 $2.147 \mathrm{~m}$. 在
2018年10月至 2019 年4月期间，海冰厚度增长速度为 $11.3 \mathrm{~cm} \mathrm{month}{ }^{-1}$, 其中 $1 \sim 4$ 月海冰厚度的增长速度达 到 $18.3 \mathrm{~cm} \mathrm{month}{ }^{-1}$.

\subsection{IceBridge海冰厚度产品验证结果}

图7为2015年3月 25 日IceBridge计划在北极中央海 域的测量航线图, 图中灰线为IceBridge航线, 黑线为当 天的CryoSat-2地面轨迹. 从图中可以发现, 在 $\mathrm{AB}$ 段两 者轨迹近乎完全重合, 其中CryoSat-2 在 $A B$ 段的工作时 间为 06:42 06:45, IceBridge在 $\mathrm{AB}$ 段的工作时间为 15:53 16:30.

利用 $\mathrm{AB}$ 段的IceBridge海冰厚度对本文计算结果 进行验证. $\mathrm{AB}$ 段全长约 $244 \mathrm{~km}$, 比较时, 将 $\mathrm{AB}$ 段每隔 $10 \mathrm{~km}$ 分为一段, 共分成 25 段, 分别计算了每段上IceBridge和CryoSat-2海冰厚度的平均值, 结果如图8所 示. 在 $\mathrm{AB}$ 段, IceBridge观测的平均海冰厚度约为 $3.077 \mathrm{~m}$, 而CryoSat-2卫星观测的平均海冰厚度为 $3.241 \mathrm{~m}$, 两者平均偏差约为 $0.165 \mathrm{~m}$.

\section{3 仰视声呐数据验证结果}

BGEP的仰视声呐观测的是海冰吃水深度, 比较时 需要转换为海冰厚度, 转换公式如下:

$h_{\mathrm{si}}=\frac{\rho_{\mathrm{sw}} h_{\mathrm{draft}}-\rho_{\mathrm{s}} h_{\mathrm{s}}}{\rho_{\mathrm{si}}}$,

式中, $h_{\text {draft }}$ 表示仰视声呐仪观测的海冰吃水深度, 海水 密度、积雪密度、海冰密度和积雪深度的取值与海冰 干舷-厚度转化中的取值相同. 仰视声呐仪的观测间隔 为 $2 \mathrm{~s}$, 比较时计算了每个月的平均海冰厚度值, 与 CryoSat-2的结果进行比较. CryoSat-2海冰厚度值为距 离仰视声呐仪 $10 \mathrm{~km}$ 范围内的平均海冰厚度.

图9分别为 $\mathrm{A} 、 \mathrm{~B} 、 \mathrm{D}$ 三个仰视声呐仪处的比较结 果, 比较时间段为2010年11月至2018年4月. 表2统计了 两者之间的最大偏差、平均偏差以及标准差. 对于仰 视声呐仪 $\mathrm{A}$, 最大偏差约为 $0.502 \mathrm{~m}$, 平均偏差约为 $0.124 \mathrm{~m}$, 标准差为 $0.133 \mathrm{~m}$; 对于仰视声呐仪 $\mathrm{B}$, 最大偏 差约为 $0.393 \mathrm{~m}$, 平均偏差约为 $0.114 \mathrm{~m}$, 标准差为 $0.094 \mathrm{~m}$; 对于仰视声呐仪 $\mathrm{D}$, 最大偏差约为 $0.354 \mathrm{~m}$, 平 均偏差约为 $0.107 \mathrm{~m}$, 标准差为 $0.115 \mathrm{~m}$. 利用CryoSat- 2 计算的海冰厚度变化趋势与三个仰视声呐仪的观测结 果基本一致. 

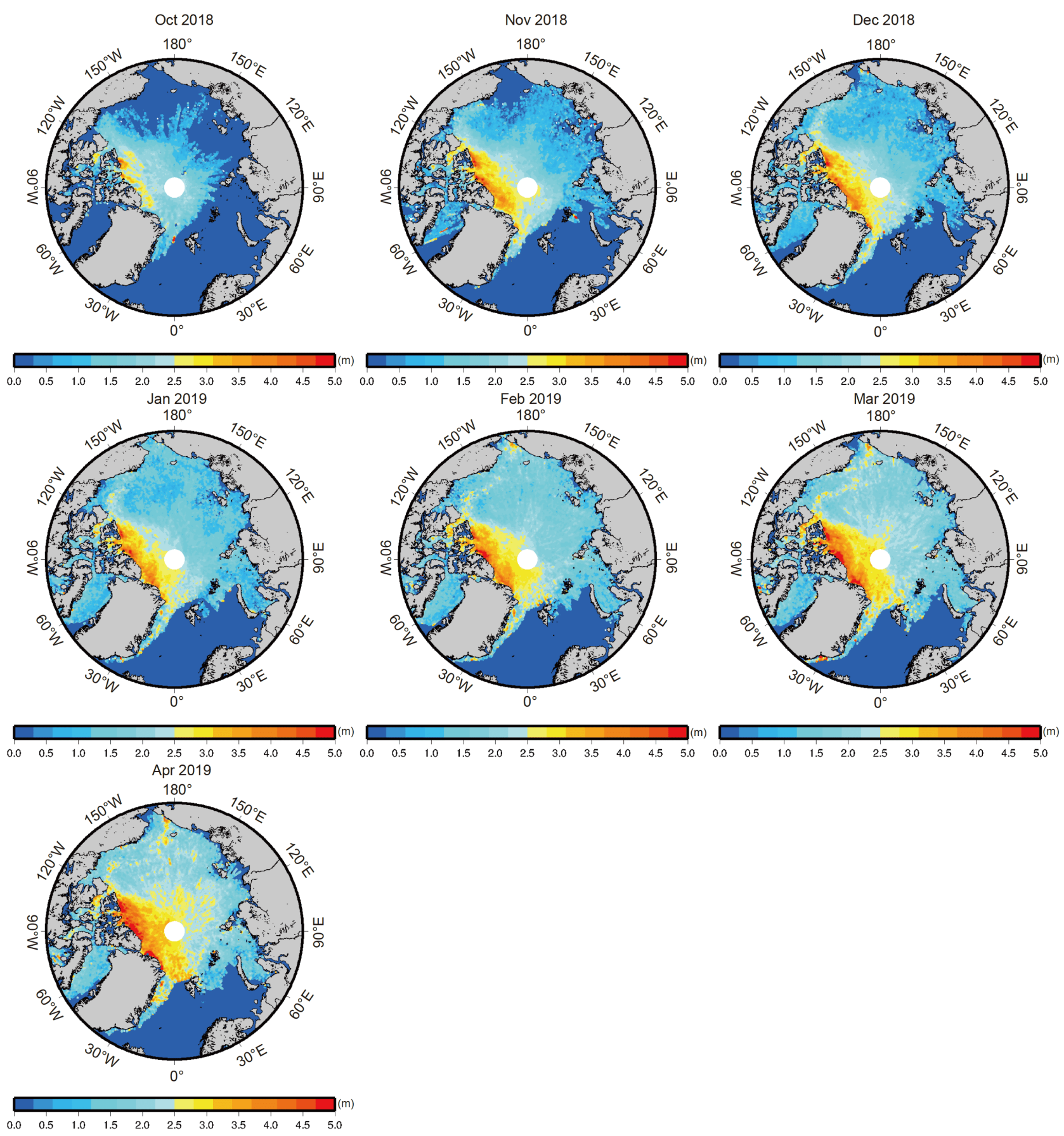

图 52018 年10月至2019年4月北极海冰厚度分布

\section{5 讨论和分析}

\section{1 北极海冰厚度年变化特征}

图10为2011 2019年期间北极海冰年平均厚度 (1 4月和10 12月)变化情况. 2012 年海冰厚度较2011
年略微下降；2013年海冰厚度增长了约 $0.040 \mathrm{~m} ; 2014$ 年海冰厚度急剧增长, 增长幅度达到 $0.165 \mathrm{~m}$; 2015 2017年, 海冰厚度持续减小, 减小速度约为 $0.035 \mathrm{~m} \mathrm{a}^{-1} ; 2018$ 年, 海冰厚度出现略微增长的现象; 2019年, 海冰厚度降低了约 $0.062 \mathrm{~m}$. 


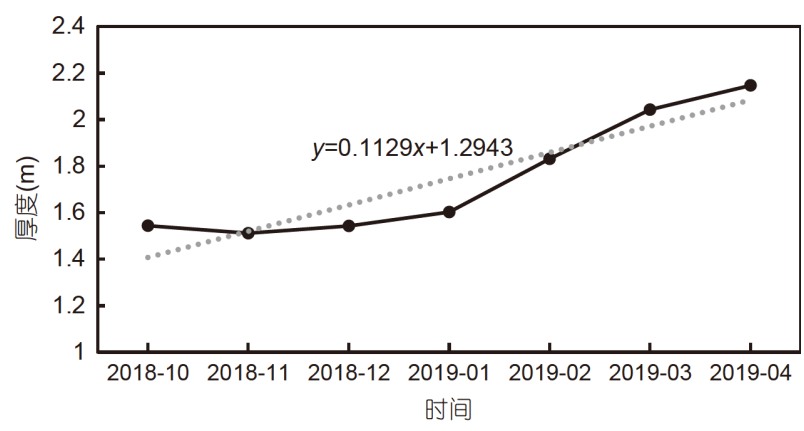

图 62018 年10月至 2019 年4月北极海冰月平均厚度变化

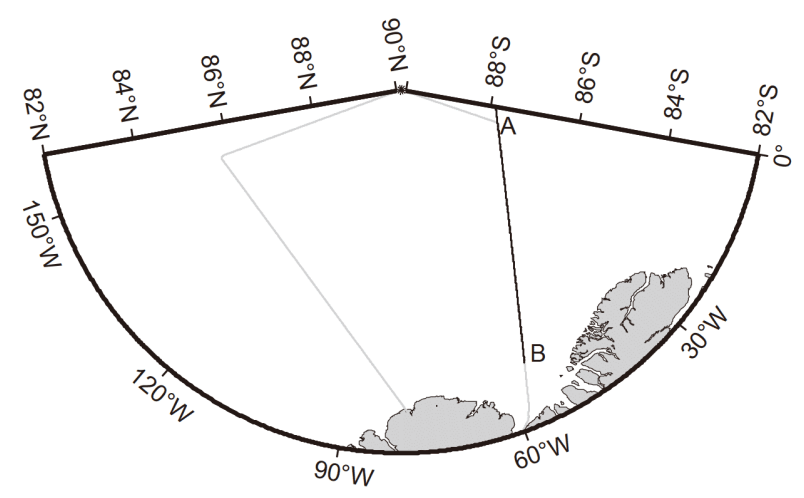

图 72015 年3月 25日 IceBridge航迹(图中灰线)与CryoSat2地面轨迹(图中黑线)

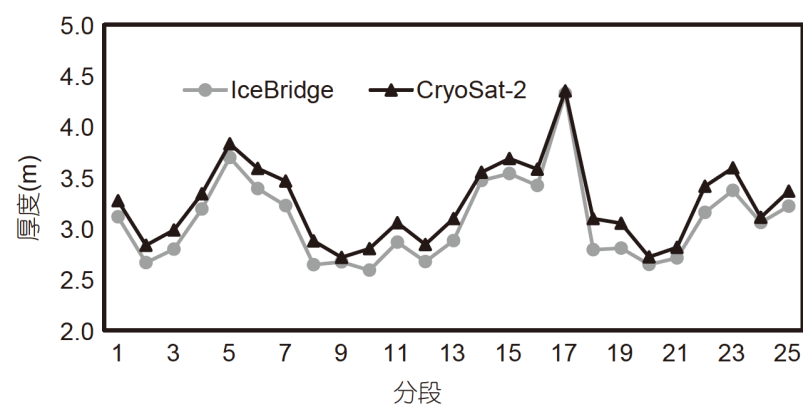

图 8 IceBridge海冰厚度与CryoSat-2海冰厚度值对比

图11为PIOMAS海冰模式给出的1979 2019年北 极海冰厚度的变化趋势. PIOMAS海冰模式(Pan-Arctic Ice Ocean Modeling and Assimilation System)是美国华 盛顿大学的Zhang和Rothrock(2003)研发的海冰模式, 该模式是全球并行海洋和海冰模式(Parallel Ocean and sea Ice Model, POIM)的组成部分(周璐等, 2017). 与其 他海冰模式不同，PIOMAS海冰模式不断同化了最新 的卫星观测的海冰信息，包括海冰密集度、厚度等.
表 2 仰视声呐仪观测的海冰厚度与CryoSat-2海冰厚度差 值统计

\begin{tabular}{lccc}
\hline & $\mathrm{A}$ & $\mathrm{B}$ & $\mathrm{D}$ \\
\hline 最大偏差 $(\mathrm{m})$ & 0.502 & 0.393 & 0.354 \\
平均偏差 $(\mathrm{m})$ & 0.124 & 0.114 & 0.107 \\
标准差 $(\mathrm{m})$ & 0.133 & 0.094 & 0.115 \\
\hline
\end{tabular}

PIOMAS海冰模式提供了自1979年至今的各种海冰参 数的估值. 为与本文计算结果一致, 图11中PIOMAS的 年平均海冰厚度为 $65^{\circ} \mathrm{N} \sim 88^{\circ} \mathrm{N}$ 范围内 $1 \sim 4$ 月和 $10 \sim 12$ 月 期间的平均厚度值. 根据PIOMAS模拟的海冰厚度结 果，自1979年来，北极海冰厚度整体上呈现减少的趋 势，其减小幅度约为 $0.020 \mathrm{~m} \mathrm{a}^{-1}$. 由图 11 可知，本文计 算的2011 2019年海冰厚度变化趋势与PIOMAS模拟 结果一致, 两者之间的平均差值约为 $0.165 \mathrm{~m}$.

图12给出了2011 2019年北极海冰区域年平均气 温(air temperature, AT)和海表面温度(sea surface temperature, SST)的变化情况. 其中气温数据来自美国国 家海洋和大气局(NOAA)提供的NCEP(National Centers for Environmental Prediction)/NCAR(National Center for Atmospheric Research)逐月再分析数据(Kalnay 等, 1996), 空间分辨率为 $2.5^{\circ} \times 2.5^{\circ}$; 海表面温度为 NOAA提供的OI(最佳插值) 逐月数据(Reynolds等, $2002)$, 空间分辨率为 $1^{\circ} \times 1^{\circ}$. 由图 12 可知, 北极海冰区 域气温和海表面温度变化基本保持同步. 2012年, 北极 气温和海表面温度较2011年上升; 2013年, 北极气温和 海表面温度突然降低；2014 2016年，北极气温和海表 面温度逐步升高; 2017 2019年, 北极气温和海表面温 度呈下降趋势．2011 2019年期间，北极气温和海表面 温度出现了两个主要拐点, 一是2013年气温和海表面 温度突然降低，与此相应的是2013年和2014年北极海 冰厚度增长, 特别是2014年海冰厚度出现剧烈增长的 现象; 另一个拐点是2017年气温和海表面温度降低的 现象，而2017年海冰厚度延续之前减小的趋势，但在 2018年海冰厚度却突然增长.

\section{2 北极海冰厚度月变化特征}

图13为2010年11月至2019年12月北极海冰月平均 厚度变化情况. 大体来看，每年10月海冰厚度值最小， 随后海冰厚度逐渐增长, 至第二年四月海冰厚度值达 到最大，此期间被认为是海冰的增长期. 表3给出了 

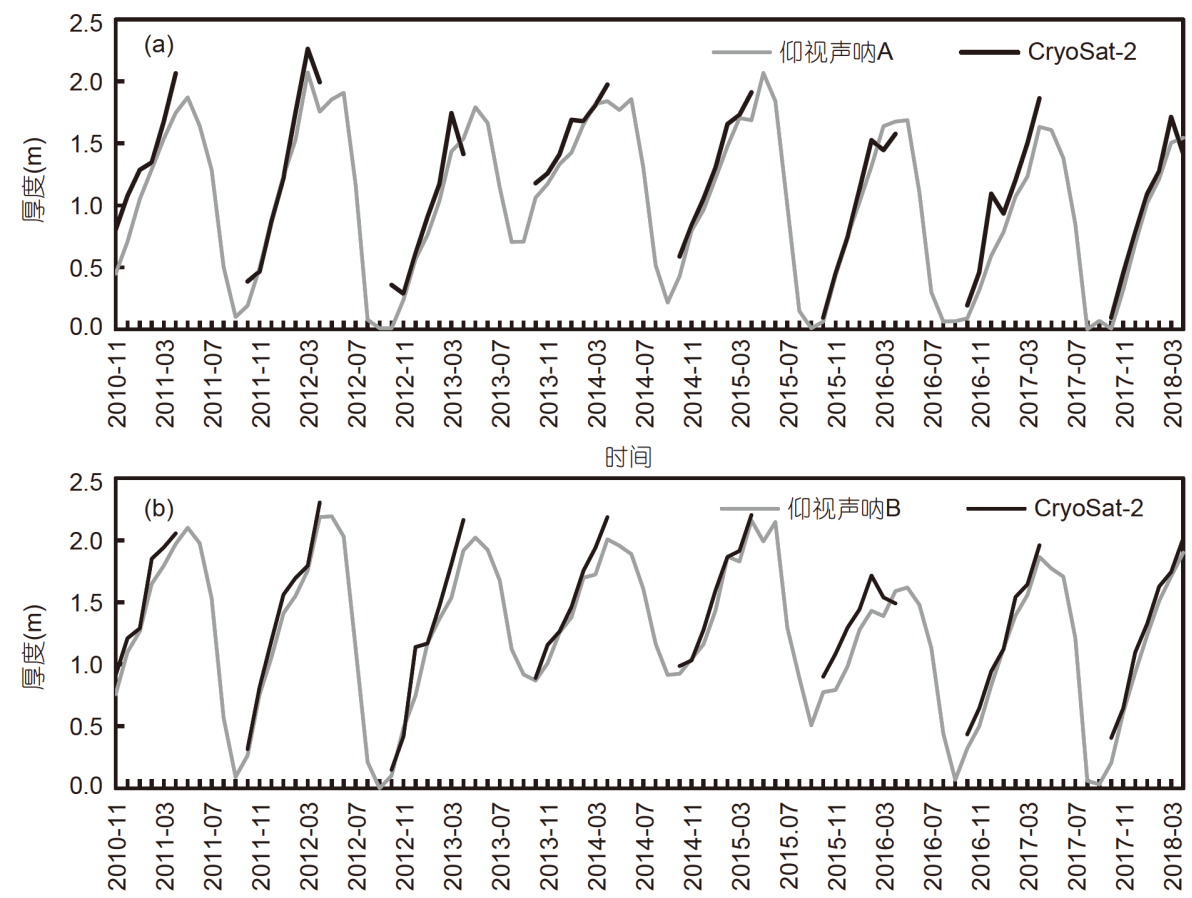

时间

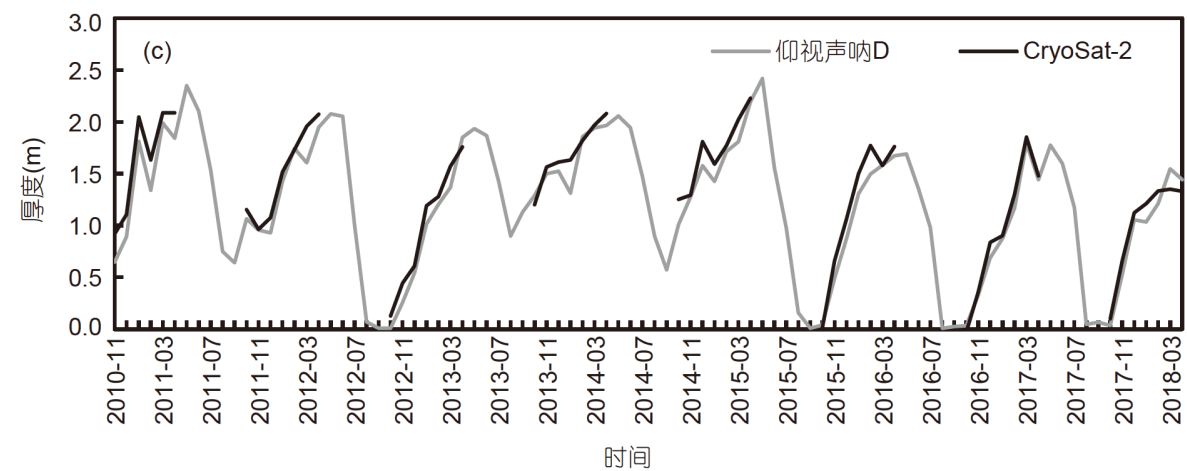

图 9 仰视声呐仪观测的海冰厚度与本文利用CryoSat-2计算的海冰厚度值对比

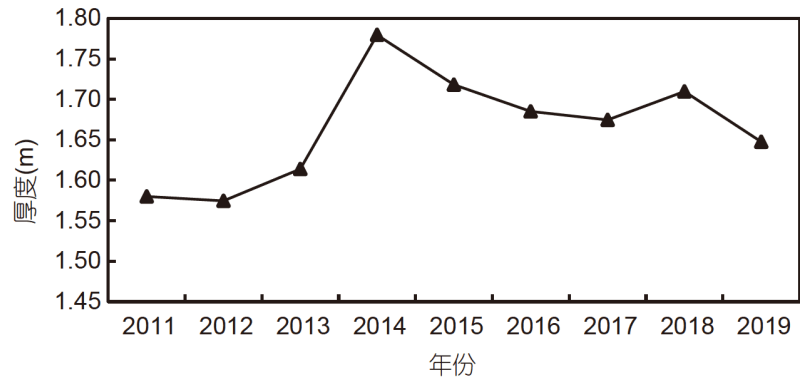

图 $10 \quad 2011 \sim 2019$ 年北极海冰年平均厚度变化

2010 2019年期间各个海冰增长期的海冰厚度增长速 度. 2010 2019年期间各个海冰增长期的海冰厚度增长

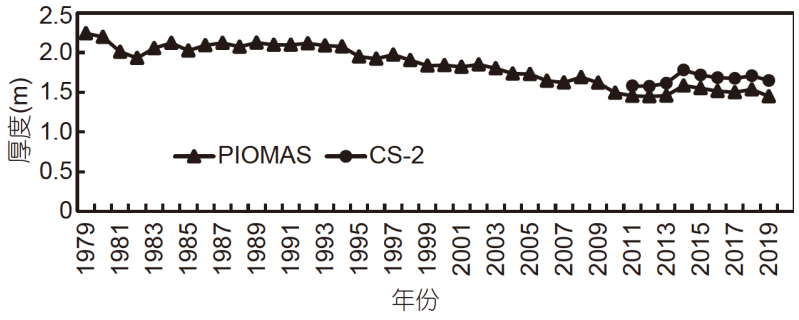

图 11 PIOMAS模式模拟的北极海冰年平均厚度变化和本 文计算结果对比

速度整体上处于降低的趋势，其中2012/2013年海冰增 长期的增长速度出现大幅度下降.

图14为2010年11月至2019年12月期间北极海冰区 


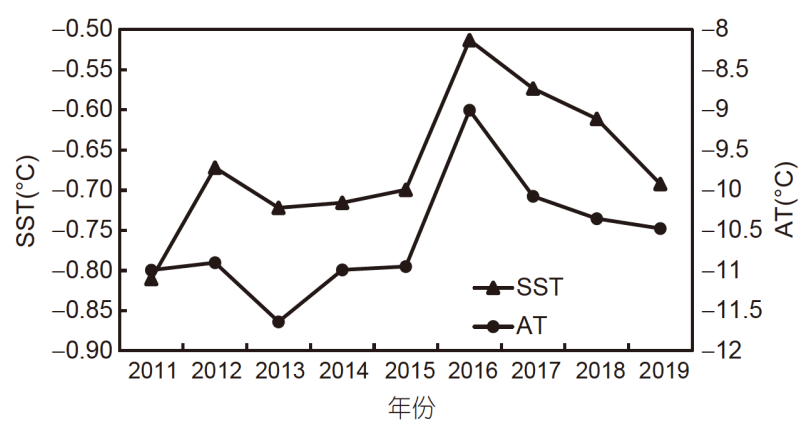

图 12 2011 2019年北极海冰区域年平均气温和海表面温 度变化

表 $32010 \sim 2019$ 年北极海冰增长期海冰厚度增长速度

\begin{tabular}{cc}
\hline 时间 & 增长速度 $\left(\mathrm{m} \mathrm{month}^{-1}\right)$ \\
\hline $2010-11 \sim 2011-04$ & 0.173 \\
$2011-10 \sim 2012-04$ & 0.165 \\
$2012-10 \sim 2013-04$ & 0.123 \\
$2013-10 \sim 2014-04$ & 0.133 \\
$2014-10 \sim 2015-04$ & 0.143 \\
$2015-10 \sim 2016-04$ & 0.134 \\
$2016-10 \sim 2017-04$ & 0.105 \\
$2017-10 \sim 2018-04$ & 0.110 \\
$2018-10 \sim 2019-04$ & 0.113 \\
\hline
\end{tabular}

月平均气温和海表面温度的变化情况. 由图14可知, $2012 / 2013$ 北极海冰增长期内平均海表面温度 $\left(-0.667^{\circ} \mathrm{C}\right)$ 高于 $2011 / 2012$ 年增长期内的平均温度值 $\left(-0.769^{\circ} \mathrm{C}\right)$, 过高的海表面温度可以认为是海冰厚度 增长速度减缓的原因之一。2013年2月北极气温出现
了骤降，同时 2013 年3月海表面温度下降幅度也超过 同期值, 与之相应的是 2013 年 3 月海冰厚度增长了 $0.283 \mathrm{~m}$ ，远远超过了 $2012 / 2013$ 年北极海冰增长期内的 增长速度 $\left(0.123 \mathrm{~m} \mathrm{month}^{-1}\right)$.

根据图 13,2013 年 10 月, 海冰厚度约为 $1.463 \mathrm{~m}$, 远 大于 2011 和 2012 年同期海冰厚度值. 图 15 给出了 2011 2019年北极海冰区域5 9月平均气温和海表面 温度变化. 2013年北极5 9月平均气温和海表面温度较 2012年同期值出现较大幅度下降，导致2013年夏季期 间海冰消融减少, 进而使得2013年10月海冰保持较大 的厚度值. 2014年北极5 9月平均气温继续降低, 使得 10 月份海冰亦保持较大的厚度值.

\section{6 结论}

本文利用CryoSat-2数据，对卫星测高反演海冰厚 度方法进行了研究，获取了2010 2019年长时间序列 的北极海冰厚度估算结果，并对其变化特征进行了分 析. 基于CryoSat-2数据, 对最低点法和波形特征法两 种冰间水道识别方法进行了对比，结果表明波形特征 方法识别效果较好. 考虑到CryoSat-2信号对海冰表面 积雪不完全穿透的特性, 通过引入雷达穿透系数, 对 海冰干舷-厚度转化模型进行优化, 计算了2010年11月 至2019年12月北极海冰厚度. 利用IceBridge海冰厚度 产品和仰视波弗特环流计划仰视声呐数据对海冰厚度 计算结果进行了验证, 结果表明: 本文的海冰厚度解算 精度约为 $0.2 \mathrm{~m}$.

结合PIOMAS海冰模式以及北极气温和海表面温

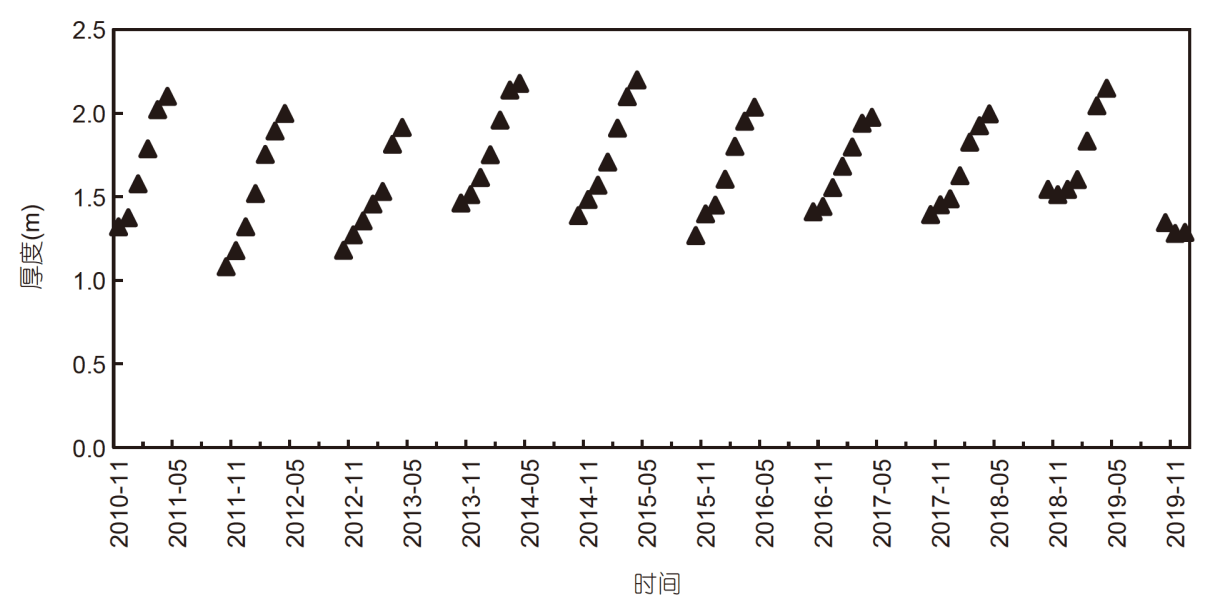

图 132010 年11月至2019年12月北极海冰月平均厚度变化 

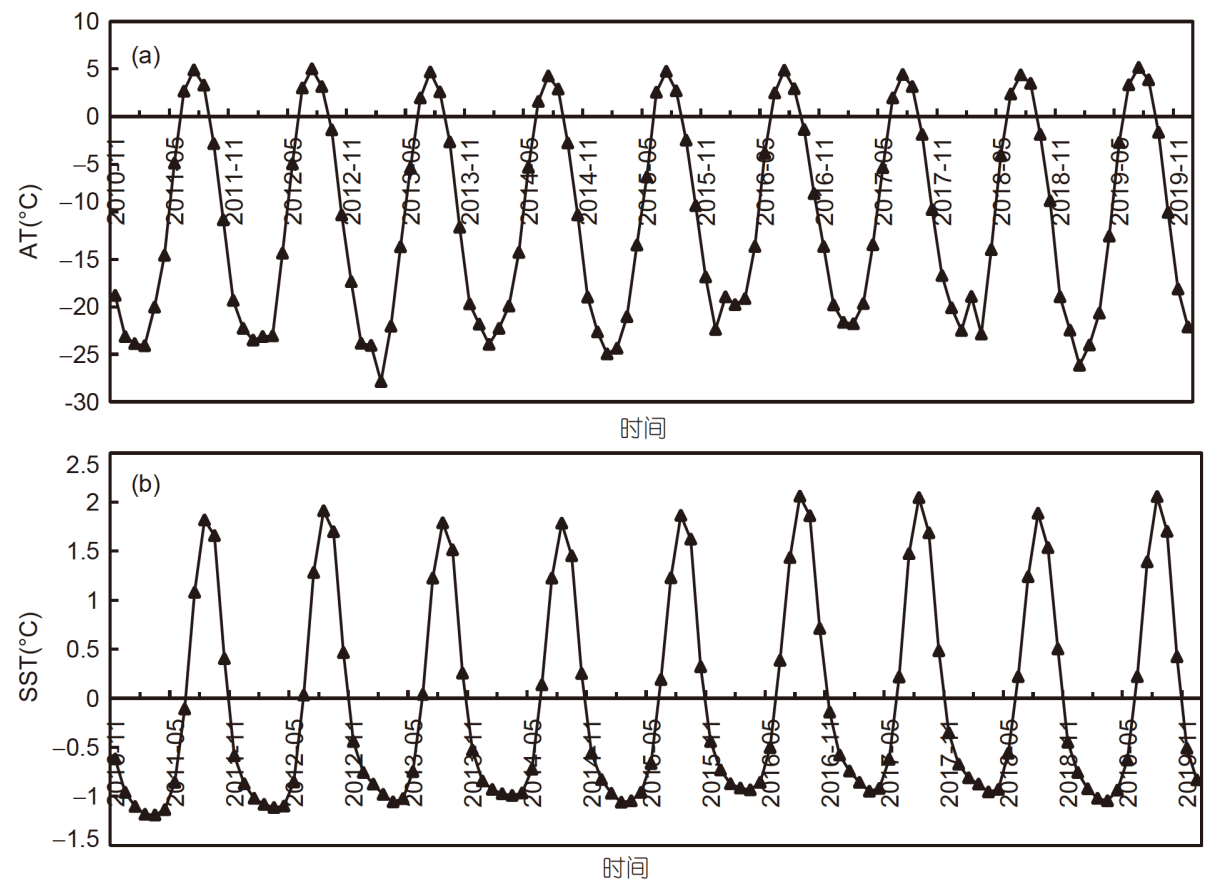

图 142010 年11月 2019年12月北极月平均气温(a)和海表面温度(b)变化

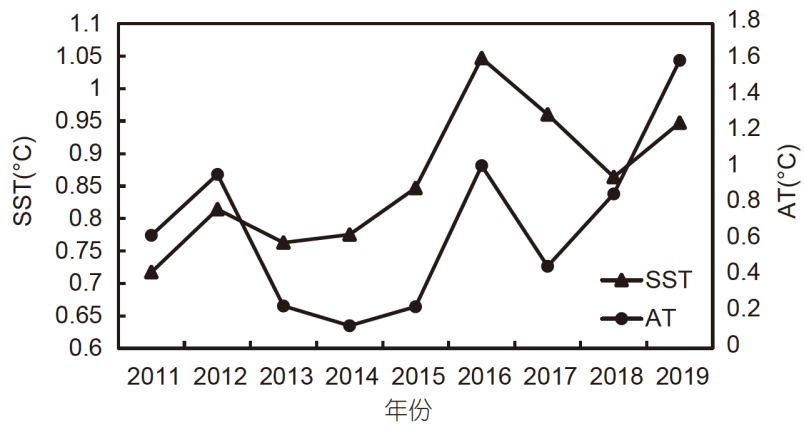

图 15 2011 2019年北极5 9月平均气温和海表面温度变化

度数据, 对北极海冰厚度变化特征进行了分析. 本文计 算的北极海冰年际变化趋势与PIOMAS模拟结果一致, 其中2014年海冰厚度出现大幅度增长. 通过对北极海 冰月平均厚度变化分析发现，北极海冰增长期主要是 每年10月至第二年4月．2010 2019年期间，北极海冰 增长期内的海冰厚度增长速度在逐渐减小.

致谢感谢欧洲空间局(ESA)提供的CryoSat-2数据, 美 国国家雪冰数据中心(NSIDC)提供的IceBridge海冰厚度 产品, 伍兹霍尔海洋学研究所提供仰视声呐数据, 美国国 家海洋和大气局(NOAA)提供的气温和海表面温度数据.

\section{参考文献}

市林根, 丁明虎, 林祥, 逯昌贵, 高志球. 2016. 北冰洋中心区夏季大 气边界层结构特征及其与海冰范围变化的关系. 中国科学: 地球 科学, 46: 555-562

秦大河, 周波涛, 效存德. 2014. 冰冻圈变化及其对中国的影响. 气象 学报, 72: 869-879

肖峰, 李斐, 张胜凯, 郝卫峰, 耿通, 宣越. 2020. 利用CryoSat-2波形数 据建立南极Lambert冰川流域DEM. 地球物理学报, 63：28932900

周璐, 徐世明, 曾刚. 2017. 20世纪90年代以来北极海冰减少的热动 力分析一一基于PIOMAS模式结果. 大气科学, 41: 57-70

Alexandrov V, Sandven S, Wahlin J, Johannessen O M. 2010. The relation between sea ice thickness and freeboard in the Arctic. Cryosphere, 4: 373-380

Armitage T W K, Ridout A L. 2015. Arctic sea ice freeboard from AltiKa and comparison with CryoSat-2 and Operation IceBridge. Geophys Res Lett, 42: 6724-6731

Breivik L A, Eastwood S, Lavergne T. 2012. Use of C-band scatterometer for sea ice edge identification. IEEE Trans Geosci Remote Sens, 50: 2669-2677

Giles K A, Laxon S W, Ridout A L. 2008. Circumpolar thinning of Arctic sea ice following the 2007 record ice extent minimum. Geophys Res Lett, 35: 2006-2009 
Kalnay E, Kanamitsu M, Kistler R, Collins W, Deaven D, Gandin L, Iredell M, Saha S, White G, Woollen J, Zhu Y, Leetmaa A, Reynolds R, Chelliah M, Ebisuzaki W, Higgins W, Janowiak J, Mo K C, Ropelewski C, Wang J, Jenne R, Joseph D. 1996. The NCEP/ NCAR 40-year reanalysis project. Bull Am Meteorol Soc, 77: 437471

Kemp J, Newhall K, Ostrom W, Krishfield R, Proshutinsky A. 2005. The Beaufort Gyre Observing System 2004: Mooring recovery and deployment operations in pack ice. WHOI Technical Report, WHOI-2005-5

Kurtz N T, Farrell S L, Studinger M, Galin N, Harbeck J P, Lindsay R, Onana V D, Panzer B, Sonntag J G. 2013. Sea ice thickness, freeboard, and snow depth products from Operation IceBridge airborne data. Cryosphere, 7: 1035-1056

Kurtz N T, Markus T, Cavalieri D J, Sparling L C, Krabill W B, Gasiewski A J, Sonntag J G. 2009. Estimation of sea ice thickness distributions through the combination of snow depth and satellite laser altimetry data. J Geophys Res, 114: 1-6

Kwok R, Cunningham G F. 2008. ICESat over Arctic sea ice: Estimation of snow depth and ice thickness. J Geophys Res, 113: C08010

Kwok R, Cunningham G F, Wensnahan M, Rigor I, Zwally H J, Yi D. 2009. Thinning and volume loss of the Arctic Ocean sea ice cover: 2003-2008. J Geophys Res, 114: 1-6

Laxon S, Peacock N, Smith D. 2003. High interannual variability of sea ice thickness in the Arctic region. Nature, 425: 947-950

Laxon S W, Giles K A, Ridout A L, Wingham D J, Willatt R, Cullen R, Kwok R, Schweiger A, Zhang J, Haas C, Hendricks S, Krishfield R, Kurtz N, Farrell S, Davidson M. 2013. CryoSat-2 estimates of Arctic sea ice thickness and volume. Geophys Res Lett, 40: 732-737

Reynolds R W, Rayner N A, Smith T M, Stokes D C, Wang W. 2002. An improved in situ and satellite SST analysis for climate. J Clim, 15: $1609-1625$

Ricker R, Hendricks S, Helm V, Skourup H, Davidson M. 2014. Sensitivity of CryoSat-2 Arctic sea-ice freeboard and thickness on radar-waveform interpretation. Cryosphere, 8: 1607-1622

Ricker R, Hendricks S, Kaleschke L, Tian-Kunze X, King J, Haas C. 2017. A weekly Arctic sea-ice thickness data record from merged CryoSat-2 and SMOS satellite data. Cryosphere, 11: 1607-1623

Screen J, Simmonds, I. 2010. The central role of diminishing sea ice in recent Arctic temperature amplification. Nature, 464: 1334-1337

Tilling R L, Ridout A, Shepherd A, Wingham D J. 2015. Increased Arctic sea ice volume after anomalously low melting in 2013. Nat Geosci, 8: 643-646

Tilling R L, Ridout A, Shepherd A. 2016. Near-real-time Arctic sea ice thickness and volume from CryoSat-2. Cryosphere, 10: 2003-2012

Tilling R L, Ridout A, Shepherd A. 2018. Estimating Arctic sea ice thickness and volume using CryoSat-2 radar altimeter data. Adv Space Res, 62: 1203-1225

Warren S G, Rigor I G, Untersteiner N, Radionov V F, Bryazgin N N, Aleksandrov Y I, Colony R. 1999. Snow depth on Arctic sea ice. J Clim, 12: 1814-1829

Willatt R, Laxon S, Giles K, Cullen R, Haas C, Helm V. 2011. Ku-band radar penetration into snow cover on Arctic sea ice using airborne data. Ann Glaciol, 52: 197-205

Wingham D J, Francis C R, Baker S, Bouzinac C, Brockley D, Cullen R, de Chateau-Thierry P, Laxon S W, Mallow U, Mavrocordatos C, Phalippou L, Ratier G, Rey L, Rostan F, Viau P, Wallis D W. 2006. CryoSat: A mission to determine the fluctuations in Earth's land and marine ice fields. Adv Space Res, 37: 841-871

Yi D, Zwally H J, Robbins J W. 2011. ICESat observations of seasonal and interannual variations of sea-ice freeboard and estimated thickness in the Weddell Sea, Antarctica (2003-2009). Ann Glaciol, 52: $43-51$

Zhang J L, Rothrock D A. 2003. Modeling global sea ice with a thickness and enthalpy distribution model in generalized curvilinear coordinates. Mon Weather Rev, 131: 845-861

Zwally H J, Yi D, Kwok R, Zhao Y. 2008. ICESat measurements of sea ice freeboard and estimates of sea ice thickness in the Weddell Sea. J Geophys Res, 113: 1-7

(责任编委: 陈大可) 